\title{
ON THE SOLUTION OF $f(f(z))=e^{z}-1$ AND ITS DOMAIN OF REGULARITY
}

\author{
ROBERT OSSERMAN
}

The problem of examining the complex solutions $f(z)$ of the equation

$$
f(f(z))=e^{z}-1
$$

was suggested to the author by S. Chowla who, together with Kempner, Rivlin, and Thron, proved that $f(z)$ cannot be an entire function. This result is contained as a special case in two recent papers $[1 ; 3]$ using the theory of entire functions. The purpose of the present note is to show how the use of elementary geometric methods leads quite easily to a slightly stronger theorem which gives some information on a maximum domain of regularity for $f(z)$. This method may also be applied to the equation

$$
f(f(z))=e^{z}
$$

which was treated in detail by Kneser [2] who proved the existence of a solution analytic on the whole real axis.

Theorem. Let $z=x+i y$ and let $\Omega$ denote an infinite strip $|y|<b$ for some constant $b>\pi$. Let $f(z)$ be a function defined in some domain $D$ such that $\Omega \subset D$ and $f(\Omega) \subset D$. If $f(z)$ satisfies (1) throughout $D$ then it cannot be analytic in $\Omega$.

Proof. Denote by $R$ the image region under $w=f(z)$ of the strip $S:|y|<\pi$. Then if $\zeta=f(w)=f(f(z))=e^{z}-1$, the image under $f(w)$ of $R$ must be the region $T$ consisting of the $\zeta$-plane slit along the negative real axis from -1 to $-\infty$. Since the composed map $\zeta=f(f(z))$ is a one-one map of $S$ onto $T$, the maps $f$ of $S$ onto $R$ and of $R$ onto $T$ must also be one-one. Furthermore, since the given correspondences between $S$ and $R$ and between $S$ and $T$ are conformal, so is the correspondence between $R$ and $T$.

We note next that $f(0)=0$. Namely, if $f(0)=a$, then $f(a)=f(f(0))$ $=0$, so that $a=f(0)=f(f(a))=e^{a}-1$. But evaluating the derivative of equation (1) at the points 0 and $a$ respectively, we find $f^{\prime}(a) f^{\prime}(0)=1$ and $f^{\prime}(0) f^{\prime}(a)=e^{a}=a+1$, whence $a=0$.

Presented to the Society, August 24, 1956 under the title On the solution of a functional equation; received by the editors March 15, 1956 and, in revised form, June 27, 1956. 
We may thus write the power expansion for $f(z)$ at the origin in the form $f(z)=a_{1} z+a_{2} z^{2}+\cdots$, and inserting this in equation (1) shows that $a_{1}=1$ and all the $a_{n}$ are real. In other words, $f^{\prime}(0)=1$ and $f(z)$ is real on the real axis. But the image of the negative $x$-axis cannot be the whole negative axis in the w-plane, since the composed map $f(f(z))$ must take the negative $x$-axis onto the interval $(-1,0)$. Hence $w=f(z)$ must map the negative $x$-axis onto an interval $(-c, 0)$ such that $f:(-c, 0) \rightarrow(-1,0)$. This implies that $c>1$.

We can now show explicitly that the function $f(z)$ must have a singularity at the point $z_{0}=\log (c-1)+i \pi$ of the region $\Omega$. To see this, denote by $R^{\prime}$ a copy of the region $R$ placed in the $\zeta$-plane. Then we can find a sequence of points $\zeta_{n}$ in the upper half $\zeta$-plane such that $\zeta_{n} \in R^{\prime} \cap T$ for all $n$, and $\zeta_{n} \rightarrow-c$. Then the corresponding points $z_{n}$ of $S$ such that $\zeta_{n}=f\left(f\left(z_{n}\right)\right)$ will approach $z_{0}$. On the other hand, their images $w_{n}=f\left(z_{n}\right)$ must approach $-\infty$. To see this we need only note that $w_{n}=f^{-1}\left(\zeta_{n}\right)$, so that if we consider the points $z_{n}^{\prime}=w_{n}$ of the $z$ plane and the points $w_{n}^{\prime}=\zeta_{n}$ of the $w$-plane, then $z_{n}^{\prime}=f^{-1}\left(w_{n}^{\prime}\right)$, and $w_{n}^{\prime} \rightarrow-c$ implies $z_{n}^{\prime} \rightarrow-\infty$.

\section{BIBLIOGRAPHY}

1. Baker, The iteration of entire transcendental functions and the solution of the functional equation $f(f(z))=F(z)$, Math. Ann. vol. 129 (1955) pp. 174-180.

2. Kneser, Reele analytische Lösungen der Gleichung $\phi(\phi(x))=e^{x}$, J. Reine Angew. Math. vol. 187 (1950) pp. 56-67.

3. Thron, Entire solutions of the functional equation $f(f(z))=g(z)$, Canadian Journal of Mathematics vol. 8 (1956) pp. 47-48.

STANFORD UnIVERSity 